\title{
Transposition of Tunneled Dysfunctional Central Catheter in Patient with Limited Vascular Access at the University Hospital of Santiago de Compostela
}

lyad Abuward Abu-sharkh ${ }^{1}$, Igor Romaniouk Jakovler ${ }^{1}$, Suleyka Puello Martinez ${ }^{1}$, Maite Rivera Gorrin ${ }^{2}$, Manuel Fidalgo ${ }^{1}$, Nisrine Arhda ${ }^{1}$, Marta Pais Seijas and Candido Díaz Rodriguez

${ }^{1}$ Department of Nephrology, Interventional and Diagnostic Nephrology Unit, University Clinical Hospital of Santiago, Spain

2Department of Nephrology, Interventional and Diagnostic Nephrology Unit, Ramon Y Cajal Hospital Madrid, Spain

*Corresponding author: Iyad Abuward Abu-sharkh, Department of Nephrology, Interventional and Diagnostic Nephrology Unit, University Clinical Hospital of Santiago, Rua de la Choupana s/n Santiago de Compostela, Spain, Tel: 0034639021641; E-mail: eyadward@hotmail.com

Received Date: July 26, 2018; Accepted Date: August 23, 2018; Published Date: August 27, 2018

Copyright: (C) 2018 Abu-sharkh I, et al. This is an open-access article distributed under the terms of the Creative Commons Attribution License, which permits unrestricted use, distribution, and reproduction in any medium, provided the original author and source are credited.

\begin{abstract}
The prevalence of chronic kidney disease in Spain has increased in recent years from 10\% of the total population affected in 2010 to $15 \%$ in 2017 according to data from the National Transplant Organization (TNO). Therefore, some of the 55,000 patients are receiving hemodialysis. Peripheral arteriovenous fistula (AVF) is the vascular access recommended by most of the clinical guides over a permanent tunneled catheter. The loss of the vascular access many times implies immediate threat to the patient's life. We present a 52-year-old patient with cardiovascular risk factors, in hemodialysis for approximately 8 years, with a long history of loss of vascular access, which presented thrombosis of the right jugular tunneled catheter in April 2017, losing the only existing access for hemodialysis, forcing to carry out a transposition of the dysfunction catheter in order to preserve the patient's life.
\end{abstract}

Keywords: Vascular access; Tunneled catheter; Hemodialysis; Endstage renal disease

\section{Case Presentation:}

A 52-year-old male patient with chronic kidney disease due to diabetic nephropathy since 2001, type 2 diabetes mellitus, arterial hypertension, hypertensive heart disease, dyslipidemia, proliferative diabetic retinopathy with retinal detachment and complete amaurosis in the left eye due to bilateral neovascular glaucoma. Vitrectomy in the right eye on March 2007. Cataract of the right eye operated in 2009. Adaptive disorder followed by psychiatry since 2007. Chronic kidney failure with hemodialysis since March of 2013. The peritoneal dialysis was ruled out due to morbid obsessiveness (body mass index 40, the patient weighed at the time of initiating dialysis $129 \mathrm{~kg}$ ).

Kidney transplant from cadaver donor August 2015 with graft dysfunction due to venous thrombosis in October. Vascular background: January 2013 starting on programmed hemodialysis using a left radiocephalic fistula with a dysfunction. February 2013 implantation of a right tunneled jugular catheter. In July 2013, a left humeral basilica prosthetic loop was performed followed by hemodialysis and removal of the right permanent catheter. In August 2015, kidney transplant of the cadaver donor was done and the loss of the graft due to venous thrombosis two month later. The patient was studied, and it is negative for the any hematological blood disorder. Prior to the end of September 2015 thrombosis of the left prosthetic loop occurred. In October 2015, prosthesis was implanted in the right iliac artery after failed graft transplantation, as well as a tunneled right jugular catheter to continue hemodialysis. In November 2015, a left humerus basilica prosthetic loop was created and the catheter was removed, continuing hemodialysis. In May of 2016, the loop fails. Vascular surgery ruled out to perform another vascular access native or prosthetic. A new tunneled catheter was implanted again in January 2017, presenting signs of catheter dysfunction three month later. After a thorough examination a subcutaneous protrusion of the catheter was seen at the base of the triangle of sedillot a phlebography (Figure 1) performed reported: thrombosis and occlusion of the right innominate vein, proximal portion of cava vein and displacement of the tunneled catheter leaving the distal end with arterial outlet in the innominatecava bifurcation. Doppler ultrasound of the femoral veins reveals a: bilateral partial thrombosis, leaving literally no vascular access for hemodialysis and putting the patients live at risk. After examination by a vascular surgeon the option offered was a creation of a prosthesis subclavian loop, being this option refused by the patient.

In order to conserve the current jugular access and trying to avoid another occlusion or the collapse of the thrombosed right innominate vein in case of the catheter removal and after checking the correct functioning with a manual reduction of the catheter angle, a transposition of the catheter was decided in order to recover its function. The procedure was done under strict asepsis rules, a resection of the fibrotic cuff-tissue block and proximal reimplantation at $4 \mathrm{~cm}$ from the original point was done, previously creating a new tunnel on top of the sterile portion of the catheter, conserving the original path. Additionally, a reintroduction of $4 \mathrm{~cm}$ of the catheter into the cava vein was done recovering a proper catheter flux at the same day of the operation (Figure 2). The patient received 14 days of antibiotic treatment with cefazoline and no signs of infection or a catheter dysfunction. 
Citation: $\quad$ Abu-sharkh IA, Jakovler IR, Martinez SP, Gorrin MR, Fidalgo M, et al. (2018) Transposition of Tunneled Dysfunctional Central Catheter in Patient with Limited Vascular Access at the University Hospital of Santiago de Compostela. J Nephrol Ther 8: 316 . doi: 10.4172/2161-0959.1000316

A

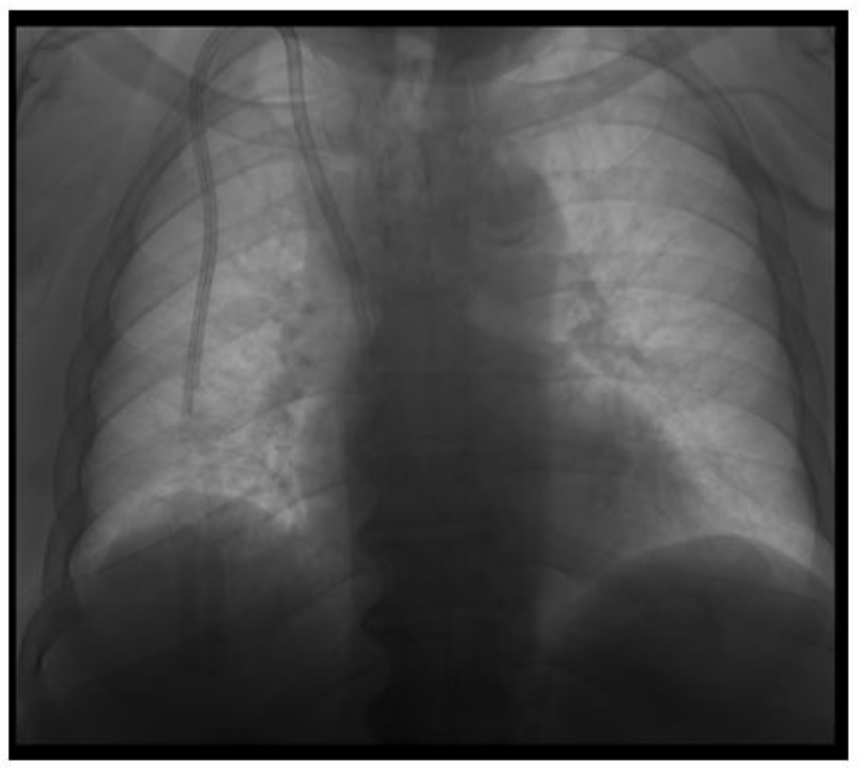

B

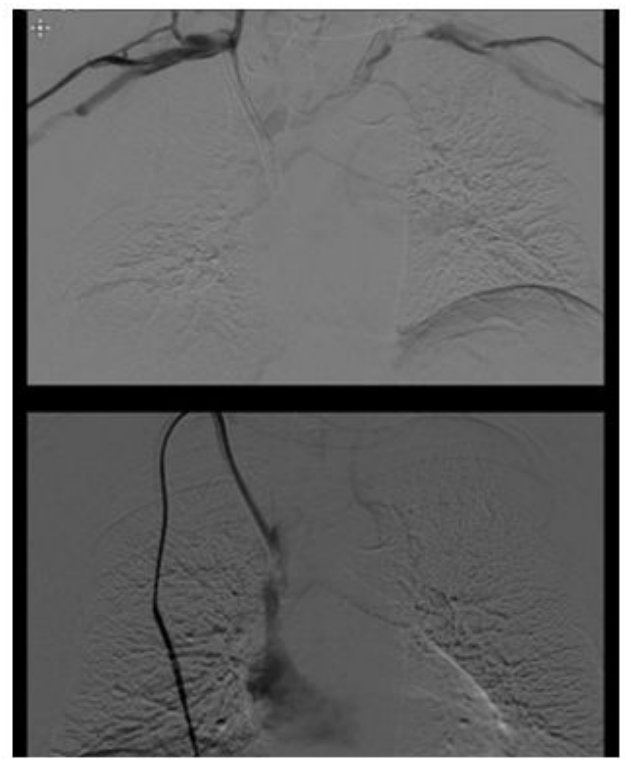

Figure 1: (A) The radiograph shows the supraclavicular protrusion of the catheter. An important thrombosis can be seen. (B) Phlebography of superior vessels that demonstrates thrombosis of both innominate veins. A trans-catheter phlebography demonstrates the permeability of catheter lumens.

A

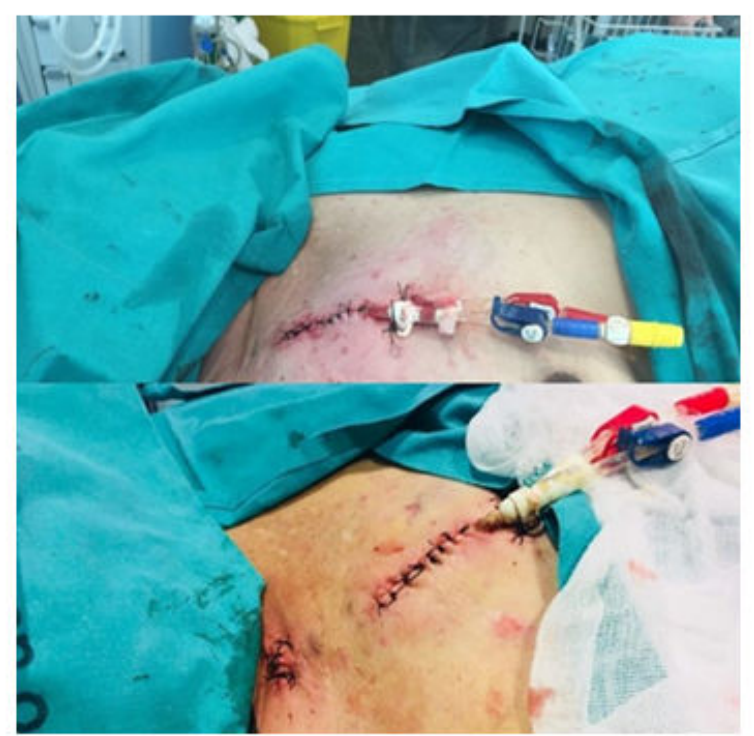

B

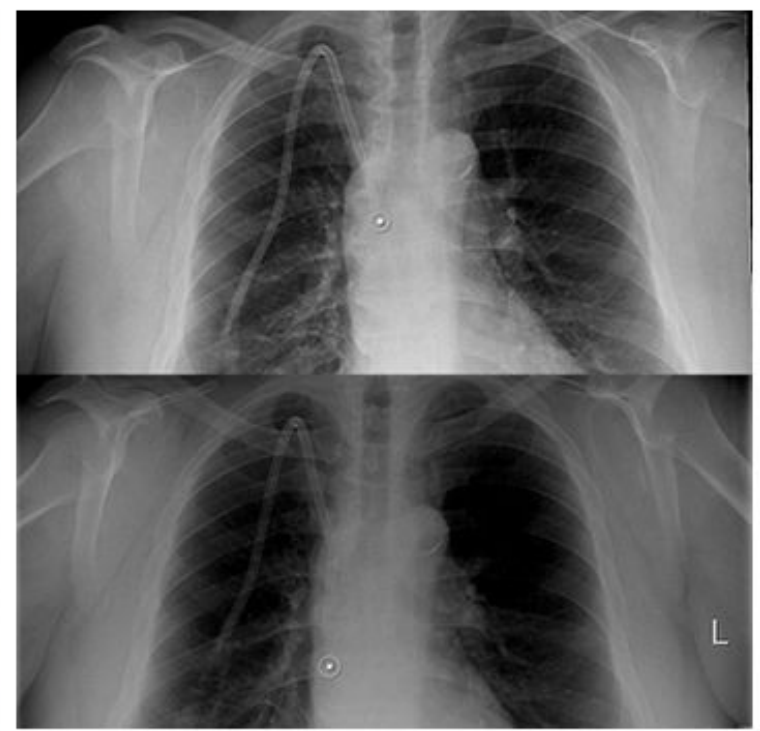

Figure 2: (A) An artificial tunnel can be seen after transposition of the cuff and the closure of superficial cervical fascia. (B) The location of the tip of the catheter before and after the transposition can be observed. 


\section{Discussion}

The number of patients who have exhausted all vascular access options will continue to increase. With different renal replacement therapy improvement, especially with the dialysis the survival rates have skyrocketed in the last ten years. That is why, the lack of vascular access is a problem that should and will increase in a future, affecting the survival of this group $[1,2]$.

The loss of vascular access in hemodialysis patients is one of the most feared complications existing. The three main types of access are: native arteriovenous fistula (AVF), arteriovenous graft, and central venous catheter $(\mathrm{CVC})$. The AVF remains the first choice for chronic HD. Other good options are prosthetic fistulae and CVCs have become an important option in HD patients. The preferable locations for insertion are the internal jugular and femoral veins. The subclavian vein is considered the third choice because of the high risk of thrombosis and stenosis $[3,4]$. Several biomarkers have been studied, such as thrombin-antithrombin (TAT), D-dimer, von Willebrand factor, PAI-1 (plasminogen activator inhibitor-1 antigen) and soluble p-selectin. A recent comparative study performed in $70 \mathrm{HD}$ patients showed that TAT, D-dimer, von Willebrand factor, p-selectin and hsCRP (high-sensitivity C-reactive protein) were all elevated in patients on HD compared with controls. In our patient, a complete study of possible coagulopathies was ruled out by hematology. However, there is another existing problem in dialysis specially with dysfunction catheters or grafts and that is an increased an increased thrombotic tendency in this group, leading not only to possibly fatal complications like ischemic heart disease or stroke, but also to thrombosis of the vascular access. The problem is even worth in polytetrafluoroethylene (PTFE) grafts. It accounts for considerable morbidity and mortality with an estimated annual cost of close to one billion United States dollars. In some cases, an underlying coagulopathy might be found. That wasn't the case in our patient, but a decreased flow due to a catheter dysfunction might lead to a further thrombosis $[5,6]$.

The most important reason for a decreasing access blood flow is intimal hyperplasia formation at the venous anastomosis or in the outflow tract of the graft. However, not all decreases in access blood flow are related to intimal hyperplasia or stenosis formation. Other causes for low access flow leading to access thrombosis have been proposed: hypovolemia, hypotension or external compression might be involved in thesenon-stenotic thrombotic events. We must remember that a normal hemostatic response is initiated by damage of the vessel wall with exposure of subendothelial structures to flowing blood and results in the formation of a solid hemostatic plug. Besides the platelets activity, fibrin is able to stabilize platelet aggregates and other cellular elements in a shear stress-resistant network. Pathological thrombin formation is prevented by natural anticoagulant systems, of which antithrombin III and the vitamin $\mathrm{K}$-dependent protein $\mathrm{C}$ systems are the most important ones. In addition to these anticoagulant systems, the fibrinolytic system generates plasmin by the action of tissue plasminogen activator on plasminogen. Plasmin dissolves the fibrin clot and thereby prevents pathological thrombus formation. Thus, normal hemostatic responses require both coagulation and platelet dependent processes. In hemodialysis patients, complex coagulation abnormalities occur, ranging from bleeding to thrombosis. On the one hand, the enhanced bleeding tendency in these patients is primarily based on functional platelet abnormalities and defective adhesion to the vessel wall. On the other hand, a variety of coagulation abnormalities contribute to an increased thrombotic tendency $[7,8]$.
Our patient had a normal coagulation time, there was no abnormality seen in intrinsic of extrinsic coagulation pathway, however other factors were involved, like transitory low blood flow due to a vascular access dysfunction and a very probable intimal hyperplasia formation at the venous anastomosis reducing the outflow tract leading to more thrombosis, more endothelium dysfunction affecting main vessels and therefore even more thrombosis.

Also, in this particular case it is important to highlight that the patient refused another prosthetic access surgery mainly due to previous prosthesis thrombosis, moreover, given the present thrombosis of the brachiocephalic vein confluence creating an access dependent of the thrombosed subclavian wasn't the best option. Therefore, other therapeutic options were considered especially those that allow preserving and somehow repairing the current vascular access. The catheter is fully functional until the present time; there is no registry yet, of another episode of thrombosis $[9,10]$.

\section{Conclusion}

We conclude that in patients with frequent vascular access complications, or with vascular access with short half-lives, or grafts, a proper care must be done to avoid early exhaustion and increase useful life. In determined sort of patients, the possibility of repairing the access even using unconventional techniques must be considered. Other factors like Hypercoagulability, hypovolemia, hypotension or external compression might be involved in these non-stenotic thrombotic events.

\section{References}

1. Ibeas J, Roca-Tey R, Vallespín J, Moreno T, Moñux G, et al. (2017) Spanish Clinical Guidelines on Vascular Access for Haemodialysis. Nefrologia 37: 1-191.

2. Martínez-Castelao A, Górriz JL, Bover J, la Morena JS, Cebollada J, et al. (2014) Consensus document for the detection and management of chronic kidney disease. Nefrología 34: 243-262.

3. Mollicone D, Pulliam J, Lacson E Jr (2013) The culture of education in a large dialysis organization: informing patient-centered decision making on treatment options for renal replacement therapy. Semin Dial 26: 143-147.

4. Pisoni RL, Zepel L, Port FK, Robinson BM (2015) Trends in US Vascular Access Use, Patient Preferences, and Related Practices: An Update From the US DOPPS Practice Monitor With International Comparisons. Am J Kidney Dis 65: 905-911.

5. Kosa SD, Al-Jaishi AA, Moist L, Lok CE (2015) Preoperative vascular access evaluation for haemodialysis patients. Cochrane Database Syst Rev $1-34$.

6. Konner K, Hulbert-Shearon TE, Roys EC, Port FK (2002) Tailoring the initial vascular access for dialysis patients. Kidney Int 62: 329-338.

7. Ravani P, Palmer SC, Oliver MJ, Quinn RR, MacRae JM, et al. (2013) Associations between hemodialysis access type and clinical outcomes: a systematic review. J Am Soc Nephrol 24: 465-473.

8. Hull JE, Makhoul RG, Snyder JF (2014) Percutaneous valvulotomy as an alternative to transposition of a brachiocephalic fistula. J Vasc Interv Radiol 25: 144-147.

9. Polo JR, Ligero JM, Diaz-Cartelle J, Garcia-Pajares R, Cervera T, et al. (2004) Randomized comparison of 6-mm straight grafts versus 6 to 8$\mathrm{mm}$ tapered grafts for brachial-axillary dialysis access. J Vasc Surg 40: 319-324.

10. Smits JH, van der Linden J, Blankestijn PJ, Rabelink TJ (2000) Coagulation and haemodialysis access thrombosis. Nephrol Dial Transplant 15: 1755-1760. 